\title{
Research on the Measurement and Spatial Correlation Analysis of Service Industry in China
}

\section{Qinglong Yang, Jian Kan}

School of Statistics and Mathematics, Zhongnan University of Economics and Law, Wuhan, 430073, China

Keywords: service industry; spatial correlation; index system

\begin{abstract}
Based on the panel data of 31 provinces in China from 2010 to 2016, the coefficient of variation method is used to construct an evaluation index system for the development level of the provincial service industry in China, and the test methods are used to analyze the spatial correlation of the development level of interprovincial service industry in China. The results show that there is a significant correlation in the development of China's service industry, and it is positively correlated, it means that the development level of a certain province's service industry has been affected by the development level of its neighboring provinces, and this effect is positive.
\end{abstract}

\section{Introduction}

In recent years, with the continuous development of global economy and information technology, service industry has gradually replaced manufacturing industry as the strongest driving force for economic growth. In China, the 19th National People's Congress pointed out that our country's economy currently is still in a critical period of structural adjustment, and should focus on developing the third industry, especially the modern service industry. However, due to the difference of economic development level between the eastern and western regions, the unbalanced development of inter-provincial service industry in China is still a hot research topic. Based on the existing results, we chooses reasonable indicators that can reflect the level of service industry development in our country, and uses the method of coefficient variation,spatial correlation test methods to research the development of the service industry between different provinces contractly,so as to provide a new research results of China's service industry development level.

\section{Test Methods}

\subsection{Spatial Correlation Test.}

Spatial autocorrelation includes global and local correlations. We use global spatial autocorrelation to verify whether there is a certain spatial relationship between service developments in provinces, and select the index to test whether the development of service industries in 31 different provinces in China is spatially related.

$$
\text { Morans' } I=\frac{\sum_{i=1}^{n} \sum_{j=1}^{n} w_{i j}\left(y_{i}-\bar{y}\right)\left(y_{j}-\bar{y}\right)}{S^{2} \sum_{i=1}^{n} \sum_{j=1}^{n} w_{i j}}
$$

Where $S^{2}=\frac{1}{n} \sum_{i=1}^{n}\left(y_{i}-\bar{y}\right)^{2}, \bar{y}=\frac{1}{n} \sum_{i=1}^{n} y_{i}$, n denotes the number of provinces in China, $\mathrm{y}_{\mathrm{j}}$ denotes the regional observations, ${ }^{w_{i j}}$ is the element in the spatial weight matrix.

\subsection{Markov Test.}

Before using the traditional Markov chain for analysis, it is necessary to verify whether the sample data has Markov properties. The inspection principle is: Utilize statistics 
$T=2 \sum_{i=1 j=1}^{k} \sum_{i j}^{k}\left[\ln \frac{Q_{i j}}{Q_{\cdot j}}\right]$,where $Q_{i j}=\frac{n_{i j}}{\sum_{j=1}^{k} n_{i j}} Q_{\cdot j}=\frac{\sum_{i=1}^{k} n_{i j}}{\sum_{i=1}^{k} \sum_{j=1}^{k} n_{i j}}$.When the sample size is large, $T$ is subject to a chi-square distribution with a degree of freedom of ${ }^{(k-1)^{2}}$. After a significant level $\alpha$ is given, if $T>\chi_{\alpha}^{2}(k-1)^{2}$, then the Markov nature of the sample data is considered, which indicates can be analyzed by the traditional Markov chain.

\section{Establishment of Service Industry Development Index System}

By sorting out relevant studies on the development level of the service industry and reconciling the current development of the service industry in China, we select 12 relevant variables from four dimensions to describe the development level of the service industry in 31 provinces of China. The index system is shown in Table 1 below:

Table 1. Index System of Development Level of Service Industry

\begin{tabular}{cll}
\hline & First-level indicators & Second-level indicators \\
\hline Development scale & GDP per capita \\
& & Output value of service industry \\
& Service investment in fixed assets \\
& The proportion of investment in services in \\
& & total investment \\
Index & & The proportion of employed population in \\
System & services in total population & The proportion of service industry in GDP \\
& Development quality & Domestic and foreign currency deposits \\
& & Integrated Cargo \\
& & Total retail sales of social consumer goods \\
& & Urbanization rate \\
& The growth rate of service industry output \\
& Industrialization rate
\end{tabular}

We choose the sample interval from 2010 to 2016, taking China's 31 provinces (excluding Tibet) as the research object and establish index system by coefficient of variation method. Taking the Beijing region as an example, the comprehensive score changes during 2010-2016 are shown in Figure 1.

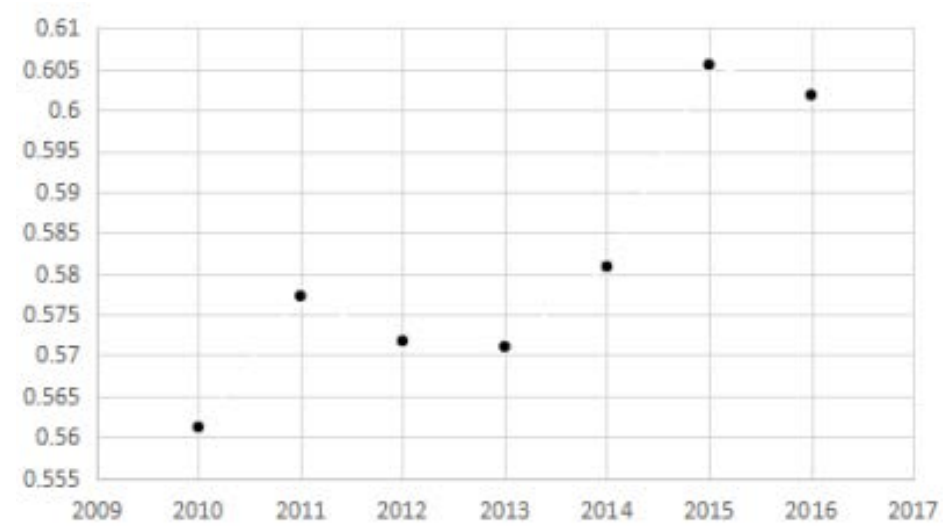

Figure 1. Overall score of the service industry in Beijing 


\section{Analysis of Markov Property and Spatial Correlation}

\subsection{Markov Analysis of Development Level of Service Industry.}

Based on the comprehensive scores of the service industry development levels of the provinces in China calculated above, we divide it into five states by using the mean-variance classification criteria' as shown in Table 2.

Table 2. Table of Service Development States

\begin{tabular}{ccc}
\hline State Number & State Level & State Range \\
\hline 1 & Low-level & $X<\bar{X}-1.5 \sigma$ \\
2 & Medium-low level & $\bar{X}-1.5 \sigma \leq X<\bar{X}-0.5 \sigma$ \\
3 & Medium-level & $\bar{X}-0.5 \sigma \leq X<\bar{X}+0.5 \sigma$ \\
4 & Medium-high level & $\bar{X}+0.5 \sigma \leq X<\bar{X}+1.5 \sigma$ \\
5 & High-level & $\bar{X}+1.5 \sigma \leq X$ \\
\hline
\end{tabular}

According to the level classification, the frequency and the probability matrix of the state transition of the service industry development level can be calculated respectively, and the Markov test statistic can be calculated: $T=352.6$. When the significant level $\alpha=0.005$ and $k=5$, the threshold is $\chi^{2}(16)=34.267$. Because $T>\chi^{2}(k-1)^{2}$, the original hypothesis is rejected,so the traditional Markov chain can be used to analyze the state transition status of China's provincial service industry, which indicates that there is a significant Markov character in the development of the service industry in various provinces. That is, the future development level of the service industry in a certain province is only related to the current development level, and is independent of the development level of the service industry in the past.

\subsection{Spatial Correlation Analysis of Development Level of Service Industry.}

In order to analyze the evolution of the development level of China's service industry from the perspective of space, it is also necessary to test the spatial correlation. According to the selected adjacency spatial weight matrix and formula (1), we calculate the global Morans'I value of the service industry development level in each region from 2010 to 2016 as shown in Table 3.

Table 3. Global Values for 2010-2016

\begin{tabular}{ccc}
\hline Year & Morans $I$ & P-value \\
\hline 2010 & 0.2733 & 0.004340 \\
2011 & 0.2521 & 0.007286 \\
2012 & 0.2713 & 0.004464 \\
2013 & 0.2494 & 0.007570 \\
2014 & 0.2864 & 0.003159 \\
2015 & 0.3138 & 0.001511 \\
2016 & 0.2777 & 0.003886 \\
\hline
\end{tabular}

From Table 3 we can see that during the period of 2010-2016, the development level of the service industry in each province in China passed the test at the $5 \%$ level of significance, and the values are all positive, which indicates that here is a significant spatial correlation between the development level of service industries in each province, and the value of the statistics is greater than zero, so the relationships of the development of the service industry is positively correlated, which indicates that for a certain province, when the development level of the service industry in its neighboring provinces is high, its own development will also be positively affected

In addition, the Morans'I value changes as the Figure 2 shows. 


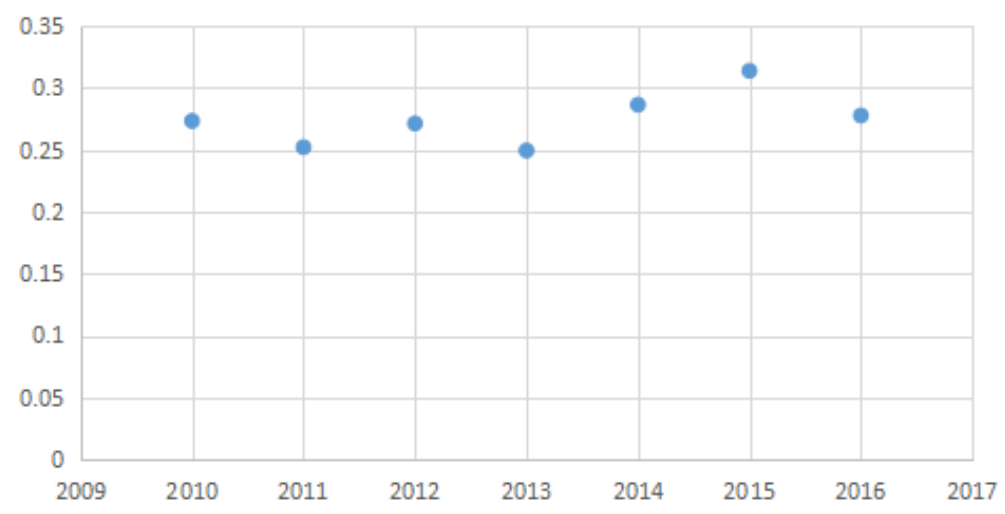

Figure 2. Morans'I value of the service industry

It can be seen from Table 2 that the spatial correlation of China's service industry development is basically between 0.25 and 0.3 in recent years, but its size fluctuations are more obvious with time. This phenomenon is not conducive to the stable development of China's overall service industry.Therefore, when the government formulate policies related to the development of the service industry, they should fully consider the synergistic development between the inter-provincial service industries to maximize the effects of this spatial correlation.

\section{Conclusion}

In our paper,we construct the service industry development level index system for the current status of 31 provinces in China from 2010 to 2016, and on the basis of index system and some relevant test methods, we find that :

(1) From the perspective of space, according to the results of the spatial correlation test, there is a significant positive correlation between the development levels of the provincial service industry. That is, the development of the service industry in a certain area will not only affect its surrounding areas, but will also be affected by its neighboring regions.

(2) From a national perspective, there is a significant Markov character in the development level of the service industry in various provinces in China, which indicates that the development level of the service industry in the future is only related to the current development level of the service industry, and is independent of the development level of the service industry in the past.

(3) In recent years, the spatial correlation fluctuation of China's service industry development has become more obvious with time. This phenomenon reflects the instability of the coordinated development of China's service industry space, and to a certain extent is not conducive to the stable development of China's overall service industry.

\section{Acknowledgement}

The paper was financially supported by National Statistical Scientific Research Project (No 2017LZ26).

\section{References}

[1] Mengyang Hou, Shunbo Yao.Measurement of Urban Eco-efficiency and Its Spatial-temporal Dynamic Evolution in China [J]. China's Population, Resources and environment, 2018 , 28(03) : 13-21.

[2] Guanghai Zhen ,Suzhen Qin. The Analysis of the Temporal and Spatial Evolution of Interprovincial Tourism Economy in China [J]. Geography and Geographical Information Science, $2014,30(02): 44-48+55$. 
[3] Li Zhou ,Shulei Xie.An Analysis of Rural Economic Development Based on Spatial Markov Chain--Taking Sichuan Province as an Example [J]. Agricultural Resources and Regional Planning in China , 2016 , 37(12) : 186-191+208.

[4] Yan H, DeChant C M, Moradkhani H. Improving soil moisture profile prediction with the particle filter-Markov chain Monte Carlo method[J]. IEEE Transactions on Geoscience and Remote Sensing, 2015, 53(11): 6134-6147.

[5] Geng Wang ,Jiali Wang et al. The Rresearch on the Spatial Evolution of Ecological Security Based on GIS-Markov Region [J]. Geographical Science , 2013 , 33(08) : 957 -964.

[6] Liang Zhang, Shijuan Guo. Weighted Markov Chain Prediction of Annual Precipitation in Zhangjiakou City[J]. Journal of Hebei Engineering and Technical College ,2014(03) : 18-21.

[7] Zhang Q, Chen Z, Yang L T. A nodes scheduling model based on Markov chain prediction for big streaming data analysis[J]. International Journal of Communication Systems, 2015, 28(9): 1610-1619.

[8] Al-sharif A A A, Pradhan B. A novel approach for predicting the spatial patterns of urban expansion by combining the chi-squared automatic integration detection decision tree, Markov chain and cellular automata models in GIS[J]. Geocarto International, 2015, 30(8): 858-881. 